\title{
Exact solutions for drying with coupled phase-change in a porous medium with a heat flux condition on the surface
}

\author{
EDUARDO A. SANTILLAN MARCUS and DOMINGO A. TARZIA \\ Depto. de Matemática and CONICET, FCE, Universidad Austral \\ Paraguay 1950, S2000FZF Rosario, Argentina \\ E-mails: Eduardo.Santillan@fce.austral.edu.ar / Domingo.Tarzia@ fce.austral.edu.ar
}

\begin{abstract}
Exact solutions for the problem of drying with coupled phase change in a porous medium with a heat flux condition on $x=0$ of the type $-q_{0} / \sqrt{\tau}$, with $q_{0}>0$, for any value of the Luikov number $L_{u}$ is obtained. This solution can be only obtained when $q_{0}$ verifies a certain inequality. Besides, for large Luikov number (more precisely, $L_{u}>\frac{1}{\varepsilon K_{0}+1}$ ), we obtain that the temperature distribution $t_{2}$ reaches to a minimum value which is smaller than its initial temperature or limit value reached at $+\infty$.
\end{abstract}

Mathematical subject classification: 35R35, 80A22, 35C 05 .

Key words: free boundary, Stefan problem, phase change, drying, heat conduction, mass transfer, porous medium.

\section{Introduction}

Heat and mass transfer with phase change problems, taking place in a porous medium, such as evaporation, condensation, freezing, melting, sublimation and desublimation, have wide application in separation processes, food technology, heat and mixture migration in soils and grounds, etc. Due to the non-linearity of the problem, solutions usually involve mathematical difficulties. Only a few exact solutions have been found for idealized cases. Mathematical formulation of the heat and mass transfer in capillary porous bodies has been established by Luikov [13], [14], [15], [16], [17]. Other problems in this direction are [3], [6], [7], [9], [20], [22]. 
A large bibliography on free and moving boundary problems for the heatdiffusion equation was given in [23]. Gupta [10] presented an approximate solution to a coupled heat and mass transfer problem involving evaporation. The problem Gupta [10] treated has analytical solution, which is presented in Cho [5].

Heat and mass transfer during drying from an homogeneous point of view are also considered in [1], [2], [4], [8], [11], [18], and [19].

In the following, we study a similar problem as that of [5]. A semi-infinite porous medium is dried by maintaining a heat flux condition at $x=0$ of the type $-q_{0} / \sqrt{t}$, with $q_{0}>0$, which was firstly considered in [21]. Initially, the whole body is at uniform temperature $t_{0}$ and uniform moisture potential $u_{0}$. The moisture is assumed to evaporate completely at a constant temperature, evaporation point $t_{v}$. It is also assumed that the moisture potential in the first region, $0<x<s(\tau)$, is constant at $u_{v}$, where $x=s(\tau)$ locates the evaporation front at time $\tau>0$. It is further assumed that the moisture in vapor form does not take away any appreciable amount of heat from the system. Neglecting mass diffusion due to temperature variation, the problem can be expressed as:

$$
\begin{gathered}
\frac{\partial t_{1}}{\partial \tau}(x, \tau)=a_{1} \frac{\partial^{2} t_{1}}{\partial x^{2}}(x, \tau), \quad 0<x<s(\tau), \tau>0 \text { (region 1) } \\
u_{1}=u_{v}, \quad 0<x<s(\tau), \tau>0 \text { (region 1) } \\
\frac{\partial t_{2}}{\partial \tau}(x, \tau)=a_{2} \frac{\partial^{2} t_{2}}{\partial x^{2}}+\frac{\varepsilon L c_{m}}{c_{2}} \frac{\partial u_{2}}{\partial \tau}, \quad x>s(\tau), \tau>0 \text { (region 2) } \\
\frac{\partial u_{2}}{\partial \tau}(x, \tau)=a_{m} \frac{\partial^{2} u_{2}}{\partial x^{2}}(x, \tau), \quad x>s(\tau), \tau>0 \text { (region 2) }
\end{gathered}
$$

The initial and boundary conditions are:

$$
\begin{gathered}
k_{1} \frac{\partial t_{1}}{\partial x}=-\frac{q_{0}}{\sqrt{\tau}} \quad \text { at } \quad x=0, \tau>0 \\
t_{2}=t_{0} \quad \text { in } \quad x>0, \tau=0 \\
u_{2}=u_{0} \quad \text { in } \quad x>0, \tau=0 \\
t_{1}(s(\tau), \tau)=t_{2}(s(\tau), \tau)=t_{v}>t_{0} \quad \text { at } \quad x=s(\tau)
\end{gathered}
$$




$$
\begin{gathered}
u_{1}(s(\tau), \tau)=u_{2}(s(\tau), \tau)=u_{v}<u_{0} \quad \text { at } \quad x=s(\tau) \\
-k_{1} \frac{\partial t_{1}}{\partial x}(s(\tau), \tau)+k_{2} \frac{\partial t_{2}}{\partial x}(s(\tau), \tau)=(1-\varepsilon) \rho_{m} L \frac{d s}{d t} \\
\text { at } x=s(\tau)
\end{gathered}
$$

Symbols are given in the nomenclature. We clarify that $t_{1}$ is the temperature of the dried porous medium, $t_{2}$ is the temperature of the humid porous medium and $u_{2}$ is the mass-transfer potential of the humid porous medium.

In paragraph 2, we find a solution of this problem, depending on the value of the Luikov number $L_{u}$, then in paragraphs 3 and 4 we discuss the equation that determines the dimensionless constant which characterizes the evaporation front when the Luikov number $L_{u}$ equals to one and $L_{u}$ is different to one. Finally, in paragraph 5 we give some illustrative results and a sufficient condition (5.4) for the Luikov number $L_{u}$ in order to obtain when the temperature distribution has a minimum value less than its initial temperature.

This study was motivated by the following mathematical and physical analysis. Taking into account (1.1), (1.5) and (1.8), and (1.4), (1.7) and (1.9), by the maximum principle, we have $t_{1}(x, \tau)>t_{v}$ for region 1 and $u_{v}<u_{2}(x, \tau)<u_{0}$ for region 2 respectively. We expect from a physical point of view that the phase change front $s(\tau)$ should be an increasing function. In this case, thanks again to the maximum principle, we should obtain that $\frac{\partial u_{2}}{\partial \tau}(x, \tau)<0$ for region 2 , then the heat equation (1.3) has a heat sink within the corresponding region 2. Due to the maximum principle, we have $t_{2}(x, \tau)<t_{v}$ for region 2 and we can say anything about where the temperature has an absolute minimum value. One of the goals of this paper is to obtain a sufficient condition for the data in order to have a minimum value for the temperature within its corresponding domain. Moreover, we can characterize the coordinate of this point when the dimensionless variable $\eta=\frac{x}{2 \sqrt{a_{1} \tau}}$ takes the value $(5.7)$ as a function of the data. 


\section{Solution of the problem}

Let be the following dimensionless variables and parameters:

$$
\begin{gathered}
U_{i}=\frac{u_{i}-u_{0}}{u_{v}-u_{0}}, \text { for } i=1,2 \\
T_{i}=\frac{t_{i}-t_{0}}{t_{v}-t_{0}}, \text { for } i=1,2 \\
\eta=\frac{x}{2 \sqrt{a_{1} \tau}} \\
L_{u}=\frac{a_{m}}{a_{1}}>0 \\
K o=\frac{L c_{m}\left(u_{0}-u_{v}\right)}{c_{2}\left(t_{v}-t_{0}\right)}>0 \\
v=\frac{(1-\varepsilon) \rho_{m} L a_{1}}{k_{1}\left(t_{v}-t_{0}\right)}>0 \\
k_{21}=\frac{k_{2}}{k_{1}}>0 .
\end{gathered}
$$

Assuming $U$ and $T$ are only functions of the variable $\eta$, the conditions (1.1)-(1.9) imply us that

$$
s(\tau)=2 \lambda \sqrt{a_{1} \tau}
$$

where $\lambda$ is a positive constant to be determined later. Therefore, equations (1.1)-(1.4) are transformed to the following dimensionless ordinary differential equations of the form:

$$
\begin{gathered}
T_{1}^{\prime \prime}(\eta)+2 \eta T_{1}^{\prime}(\eta)=0, \quad 0<\eta<\lambda \\
U_{1}=1, \quad 0<\eta<\lambda \\
T_{2}^{\prime \prime}(\eta)+2 \eta T_{2}^{\prime}(\eta)-2 \varepsilon K_{o} \eta U_{2}^{\prime}(\eta)=0, \quad \eta>\lambda \\
L_{u} U_{2}^{\prime \prime}(\eta)+2 \eta U_{2}^{\prime}(\eta)=0, \quad \eta>\lambda .
\end{gathered}
$$


The boundary conditions (1.5)-(1.10) become:

$$
\begin{gathered}
T_{1}^{\prime}=-\frac{2 q_{0} \sqrt{a_{1}}}{k_{1}\left(t_{v}-t_{0}\right)} \text { at } \eta=0, \\
T_{2}=0 \text { as } \eta \rightarrow \infty, \\
U_{2}=0 \quad \text { as } \quad \eta \rightarrow \infty, \\
T_{1}=T_{2}=1 \quad \text { at } \quad \eta=\lambda, \\
U_{1}=U_{2}=1 \quad \text { at } \quad \eta=\lambda, \\
T_{1}^{\prime}-k_{21} T_{2}^{\prime}=-2 v \lambda \quad \text { at } \eta=\lambda,
\end{gathered}
$$

Solutions of the equations (2.9) and (2.12), which satisfy boundary conditions (2.13), (2.15), (2.16) and (2.17), are easily obtained as follows

$$
\begin{gathered}
T_{1}(\eta)=1+\frac{q_{0} \sqrt{\pi a_{1}}}{k_{1}\left(t_{v}-t_{0}\right)}(\operatorname{erf} \lambda-\operatorname{erf} \eta), \quad 0<\eta<\lambda \\
U_{2}(\eta)=\frac{1-\operatorname{erf}\left(\frac{\eta}{\sqrt{L_{u}}}\right)}{1-\operatorname{erf}\left(\frac{\lambda}{\sqrt{L_{u}}}\right)}, \quad \eta>\lambda .
\end{gathered}
$$

Substituting expression (2.20) into equation (2.11), and solving the resulting non-homogeneous ordinary differential equation with boundary conditions (2.14) and (2.16), we obtain the following results, depending on $L_{u}=1$ or $L_{u} \neq 1$, i.e.:

$$
\begin{aligned}
T_{2}(\eta)= & \frac{\varepsilon K_{o}}{\sqrt{\pi}(1-\operatorname{erf}(\lambda))}\left[\lambda e^{-\lambda^{2}} \frac{1-\operatorname{erf}(\eta)}{1-\operatorname{erf}(\lambda)}-\eta e^{-\eta^{2}}\right] \\
& +\frac{1-\operatorname{erf}(\eta)}{1-\operatorname{erf}(\lambda)}, \quad \text { if } \quad L_{u}=1, \eta>\lambda
\end{aligned}
$$


or

$$
\begin{aligned}
T_{2}(\eta)= & \frac{\varepsilon K_{o} L_{u}}{L_{u}-1}\left[-\frac{1-\operatorname{erf}\left(\frac{\eta}{\sqrt{L_{u}}}\right)}{1-\operatorname{erf}\left(\frac{\lambda}{\sqrt{L_{u}}}\right)}+\frac{1-\operatorname{erf}(\eta)}{1-\operatorname{erf}(\lambda)}\right] \\
& +\frac{1-\operatorname{erf} \eta}{1-\operatorname{erf} \lambda}, \quad \text { if } L_{u} \neq 1, \eta>\lambda .
\end{aligned}
$$

Functions (2.19), (2.20) and (2.21) or (2.22) satisfy all boundary conditions except condition (2.18). Substituting these expressions into condition (2.18), the positive constant $\lambda$ is determined from the following equation, depending on the value of $L_{u}$, as follows:

$$
\begin{gathered}
\frac{k_{21}}{\sqrt{\pi}} \frac{e^{-\lambda^{2}}}{1-\operatorname{erf}(\lambda)}\left[-\frac{2 \varepsilon K_{0}}{\sqrt{\pi}} \lambda \frac{e^{-\lambda^{2}}}{1-\operatorname{erf}(\lambda)}+2 \varepsilon K_{0} \lambda^{2}-\varepsilon K_{0}-2\right] \\
+\frac{2 \sqrt{a_{1}} q_{0}}{k_{1}\left(t_{v}-t_{0}\right)} e^{-\lambda^{2}}=2 v \lambda, \quad \lambda>0 \quad \text { if } L_{u}=1,
\end{gathered}
$$

or

$$
\begin{gathered}
\frac{\sqrt{\pi a_{1}} q_{0}}{\left(t_{v}-t_{0}\right)} e^{-\lambda^{2}}+\frac{L_{u} \varepsilon K_{0}}{L_{u}-1} k_{2}\left[\frac{1}{\sqrt{L_{u}}} F_{1}\left(\frac{\lambda}{\sqrt{L_{u}}}\right)-F_{1}(\lambda)\right] \\
=k_{2} F_{1}(\lambda)+\sqrt{\pi} k_{1} v \lambda, \quad \lambda>0 \quad \text { if } L_{u} \neq 1 .
\end{gathered}
$$

\section{Discussion of the equation that determines $\lambda$, considering the case when the Luikov number equals to one}

Now let's study in detail the equation (2.23), vinculated to the case $L_{u}=1$, that is to say, when $a_{m}=a_{1}$. We define the following real functions:

$$
\begin{aligned}
\alpha(x)= & \frac{k_{21}}{\sqrt{\pi}} \frac{e^{-x^{2}}}{1-\operatorname{erf}(x)}\left[-\frac{2 \varepsilon K_{0}}{\sqrt{\pi}} x \frac{e^{-x^{2}}}{1-\operatorname{erf}(x)}+2 \varepsilon K_{0} x^{2}-\varepsilon K_{0}-2\right] \\
& +\frac{2 \sqrt{a_{1}} q_{0}}{k_{1}\left(t_{v}-t_{0}\right)} e^{-x^{2}}
\end{aligned}
$$


Then, equation (2.23) can be expressed saying that $\lambda$ must be the solution of the following equation

$$
\alpha(x)=\chi(x), \quad x>0 .
$$

We shall see the characteristics of each one of the functions $\alpha$ and $\chi$ which appears in equation (3.3).

Firstly, we have that $\chi$ is a strictly increasing function, with the properties:

$$
\chi(0)=0 ; \quad \chi(+\infty)=+\infty ; \quad \chi^{\prime}(x)=2 v>0, x>0 .
$$

Before we study the function $\alpha$, let's define the following real functions:

$$
\begin{gathered}
Q(x)=\sqrt{\pi} x e^{-x^{2}}(1-\operatorname{erf}(x)), \quad x>0 \\
W(x)=\frac{x}{\sqrt{\pi}} \frac{e^{-x^{2}}}{1-\operatorname{erf}(x)}-x^{2}=x^{2}\left(\frac{1}{Q(x)}-1\right), \quad x>0 .
\end{gathered}
$$

Function $Q$ has the following properties:

$$
Q\left(0^{+}\right)=0 ; \quad Q(+\infty)=1 ; \quad Q^{\prime}(x)>0, x>0 .
$$

Function $W$ is a positive valued function, with the following properties [12]:

$$
W\left(0^{+}\right)=0 ; \quad W(+\infty)=\frac{1}{2} ; \quad W^{\prime}(x)>0
$$

then $W$ is a strictly increasing function. Now we take care about $\alpha$. Taking into account $W$, we can put $\alpha$ in the following way:

$$
\alpha(x)=\frac{2 \sqrt{a_{1}} q_{0}}{k_{1}\left(t_{v}-t_{0}\right)} e^{-x^{2}}-\frac{k_{21}}{\sqrt{\pi}} F_{1}(x)\left[2 \varepsilon K_{0} W(x)+\varepsilon K_{0}+2\right]
$$

where function $F_{1}$ is defined by

$$
F_{1}(x)=\frac{e^{-x^{2}}}{1-\operatorname{erf}(x)}
$$

which has the following properties

$$
F_{1}\left(0^{+}\right)=1 ; \quad F_{1}(+\infty)=+\infty ; \quad F_{1}^{\prime}(x)>0, x>0 ; \quad F_{1}^{\prime \prime}(x)>0, x>0
$$




$$
\lim _{x \rightarrow+\infty} \frac{F_{1}(x)}{x}=\frac{\sqrt{\pi}}{Q(+\infty)}=\sqrt{\pi} .
$$

Then $\alpha$ is written as the sum of two strictly decreasing functions, therefore it results that $\alpha$ is also a strictly decreasing one. Besides, it has the following properties:

$$
\begin{aligned}
\alpha(0)= & \frac{2 \sqrt{a_{1}} q_{0}}{k_{1}\left(t_{v}-t_{0}\right)}-\frac{k_{21}}{\sqrt{\pi}}\left[\varepsilon K_{0}+2\right] ; \quad \alpha(+\infty)=-\infty \\
\alpha^{\prime}(x)= & \frac{-4 x \sqrt{a_{1}} q_{0}}{k_{1}\left(t_{v}-t_{0}\right)} e^{-x^{2}}-\frac{k_{21}}{\sqrt{\pi}} \frac{e^{-x^{2}}}{1-\operatorname{erf}(x)}\left[2 \varepsilon K_{0} W^{\prime}(x)\right] \\
& +\frac{\left(-k_{21}\right)}{\sqrt{\pi}} F_{1}^{\prime}(x)\left[2 \varepsilon K_{0} W(x)+\varepsilon K_{0}+2\right]<0, \quad x>0 .
\end{aligned}
$$

Next, to assure that the two functions $\alpha$ and $\chi$ have an intersection point, we need to assume that

$$
\alpha(0)>\chi(0),
$$

that is to say, $\frac{2 \sqrt{a_{1}} q_{0}}{k_{1}\left(t_{v}-t_{0}\right)}>\frac{k_{21}}{\sqrt{\pi}}\left[\varepsilon K_{0}+2\right]$, which is equivalent to the condition

$$
q_{0}>\frac{k_{2}\left(t_{v}-t_{0}\right)}{2 \sqrt{\pi a_{1}}}\left[\varepsilon K_{0}+2\right],
$$

and we can finally give the following:

Theorem 3.1. If the Luikov number is equals to one, and the coefficient $q_{0}$ verifies the condition (3.5) then there exists one and only one solution $\lambda>0$ of the equation (2.23). Furthermore, the solution of the problem (1.1)-(1.10) is given by (2.19)-(2.21), where $\lambda$ is the unique solution of the equation (2.23), that is:

$$
\begin{gathered}
u_{1}(x, \tau)=u_{v}, \quad 0<x<s(\tau), \tau>0 \\
t_{1}(x, \tau)=1+\frac{q_{0} \sqrt{\pi a_{1}}}{k_{1}\left(t_{v}-t_{0}\right)}\left(\operatorname{erf} \lambda-\operatorname{erf}\left(\frac{x}{2 \sqrt{a_{1} \tau}}\right)\right), \\
0<x<s(\tau), \tau>0
\end{gathered}
$$




$$
\begin{aligned}
u_{2}(x, \tau)=\frac{1-\operatorname{erf}\left(\frac{x}{2 \sqrt{a_{m} \tau}}\right)}{1-\operatorname{erf}(\lambda)}, \quad x>s(\tau), \tau>0 \\
t_{2}(\eta)=\frac{\varepsilon K_{0}}{\sqrt{\pi}(1-\operatorname{erf}(\lambda))}\left[\lambda e^{-\lambda^{2}} \frac{1-\operatorname{erf}\left(\frac{x}{2 \sqrt{a_{1} \tau}}\right)}{1-\operatorname{erf}(\lambda)}-\frac{x}{2 \sqrt{a_{1} \tau}} e^{-\frac{x^{2}}{4 a_{1} \tau}}\right] \\
+\frac{1-\operatorname{erf}\left(\frac{x}{2 \sqrt{a_{1} \tau}}\right)}{1-\operatorname{erf} \lambda}, \quad x>s(\tau), \tau>0 \\
s(\tau)=2 \lambda \sqrt{a_{1} \tau}
\end{aligned}
$$

\section{Discussion of the equation that determines $\lambda$, considering the case when the Luikov number is different to one}

In this paragraph we will study in detail the equation (2.24), which determines the unknown $\lambda$ for the case $L_{u} \neq 1$, that is to say, $a_{m} \neq a_{1}$. For this propose, we define the following functions:

$$
\begin{aligned}
& \phi(x)=\frac{\sqrt{\pi a_{1}} q_{0}}{\left(t_{v}-t_{0}\right)} e^{-x^{2}}+P(x) \\
& \varphi(x)=k_{2} F_{1}(x)+\sqrt{\pi} k_{1} v x .
\end{aligned}
$$

where

$$
P(x)=\frac{L_{u} \varepsilon K_{0}}{L_{u}-1} k_{2}\left(\frac{1}{\sqrt{L_{u}}} F_{1}\left(\frac{x}{\sqrt{L_{u}}}\right)-F_{1}(x)\right), \quad x>0 .
$$

Then, equation (2.24) can be written saying that $\lambda$ must be the solution of the equation

$$
\phi(x)=\varphi(x), \quad x>0
$$

Therefore, we can see the characteristics of each one of these functions $\phi$ and $\varphi$. 
Firstly, let's see that $\varphi(x)$ is a strictly increasing function with the properties:

$$
\varphi(0)=k_{2} ; \quad \varphi(+\infty)=+\infty ; \quad \varphi^{\prime}(x)>0, x>0 .
$$

Before studying $\phi$, we need to analyse the function $P$.

Obviously, function $P$ when $x=0^{+}$is equal to $-\frac{\sqrt{L_{u}} \varepsilon K_{0}}{\sqrt{L_{u}}+1} k_{2}<0$, and when $x$ tends to $+\infty$ its behaviour may depends on the value of $L_{u}$. Well, it doesn't happen:

We have

$$
\lim _{x \rightarrow \infty}\left(\frac{F_{1}\left(\frac{x}{\sqrt{L_{u}}}\right)}{F_{1}(x)}-\sqrt{L_{u}}\right)=\frac{1}{\sqrt{L_{u}}}-\sqrt{L_{u}}=\frac{1-L_{u}}{\sqrt{L_{u}}},
$$

so, we can verify that:

i) If $L_{u}>1$, we have $\lim _{x \rightarrow \infty}\left(\frac{1}{\sqrt{L_{u}}} F_{1}\left(\frac{x}{\sqrt{L_{u}}}\right)-F_{1}(x)\right)=-\infty$, then $P(+\infty)=-\infty$.

ii) If $L_{u}<1$, we have $\lim _{x \rightarrow \infty}\left(\frac{1}{\sqrt{L_{u}}} F_{1}\left(\frac{x}{\sqrt{L_{u}}}\right)-F_{1}(x)\right)=+\infty$, then $P(+\infty)=-\infty$.

Therefore, it doesn't matter whether $L_{u}$ is less or greater than 1, $P(x)$ always tends to $-\infty$ when $x \rightarrow+\infty$. Then, the properties of $\phi(x)$ are:

$$
\begin{gathered}
\phi(0)=\frac{\sqrt{\pi a_{1}} q_{0}}{\left(t_{v}-t_{0}\right)}+P\left(0^{+}\right)=\frac{\sqrt{\pi a_{1}} q_{0}}{\left(t_{v}-t_{0}\right)}-\frac{\sqrt{L_{u}} \varepsilon K_{0}}{\sqrt{L_{u}}+1} k_{2} ; \phi(+\infty)=-\infty \\
\phi^{\prime}(x)=-\frac{2 \sqrt{\pi a_{1}} q_{0}}{\left(t_{v}-t_{0}\right)} x e^{-x^{2}}+\frac{L_{u} \varepsilon K_{0}}{L_{u}-1} k_{2}\left[\frac{1}{L_{u}} F_{1}^{\prime}\left(\frac{x}{\sqrt{L_{u}}}\right)-F_{1}^{\prime}(x)\right]<0, x>0 .
\end{gathered}
$$

Concluding, to assure an intersection point between the two functions $\phi$ and $\varphi$, we impose the condition $\phi(0)>\varphi(0)$, that is to say

$$
\frac{\sqrt{\pi a_{1}} q_{0}}{\left(t_{v}-t_{0}\right)}-\frac{\sqrt{L_{u}} \varepsilon K_{0}}{\sqrt{L_{u}}+1} k_{2}>k_{2}
$$

which is equivalent to

$$
q_{0}>k_{2}\left(1+\frac{\sqrt{L_{u}} \varepsilon K_{0}}{1+\sqrt{L_{u}}}\right) \frac{t_{v}-t_{0}}{\sqrt{\pi a_{1}}},
$$

and we can finally give the following theorem: 
Theorem 4.1. If the Luikov number is different than one, and the coefficient $q_{0}$ verifies the condition (4.5) then there exists one and only one solution $\lambda>0$ of the equation (2.24). Furthermore, the solution of the problem (1.1)-(1.10) is given by (2.19)-(2.21), where $\lambda$ is the solution of the equation (2.24), that is: (3.6), (3.7), (3.8), (3.10) and

$$
\begin{aligned}
t_{2}(\eta)= & \frac{\varepsilon K_{o} L_{u}}{L_{u}-1}\left[-\frac{1-\operatorname{erf}\left(\frac{x}{2 \sqrt{a_{m} \tau}}\right)}{1-\operatorname{erf}\left(\frac{\lambda}{\sqrt{L_{u}}}\right)}+\frac{1-\operatorname{erf}\left(\frac{x}{2 \sqrt{a_{1} \tau}}\right)}{1-\operatorname{erf}(\lambda)}\right] \\
& +\frac{1-\operatorname{erf}\left(\frac{x}{2 \sqrt{a_{1} \tau}}\right)}{1-\operatorname{erf} \lambda}, \quad x>s(\tau), \tau>0 .
\end{aligned}
$$

Remark 1. The right side member of the inequality (4.5) goes to the right side member of the inequality (3.5) when $L_{u}$ tends to 1 , that is to say, we can study the case $L_{u}=1$ considering the limit $L_{u} \rightarrow 1$ in the case $L_{u} \neq 1$, then we can resume both results in the following one:

Theorem 4.2. Let be consider the coefficient $q_{0}$ verifying the condition (4.5), then, for any positive value of $L_{u}$, there exists one and only one solution $\lambda>0$ of the equation (2.23) or (2.24) depending on what value takes $L_{u}$. Furthermore, the solution of the problem (1.1)-(1.10) is given by:

a) (3.7)-(3.8), (3.9) and (3.10), if $L_{u}=1$,

b) (3.7)-(3.8), (4.6) and (3.10), if $L_{u} \neq 1$.

\section{Some illustrative results and a sufficient condition for the Luikov number in order to obtain the minimum value of the temperature distribution}

Some results of sample calculations are shown here. In this examples we take $\varepsilon K_{0}=2, a_{1}=1, k_{2}=1$, and $\left(t_{v}-t_{0}\right)=1$. Figure 1 shows the behaviour of $\lambda$ as a function of $q_{0}$. Figure 2, 3 and 4 shows the behaviour of the dimensionless temperature with respect to the dimensionless variable $\eta$, taking $L_{u}$ equals to $0.1,1$ and 4 respectively. 


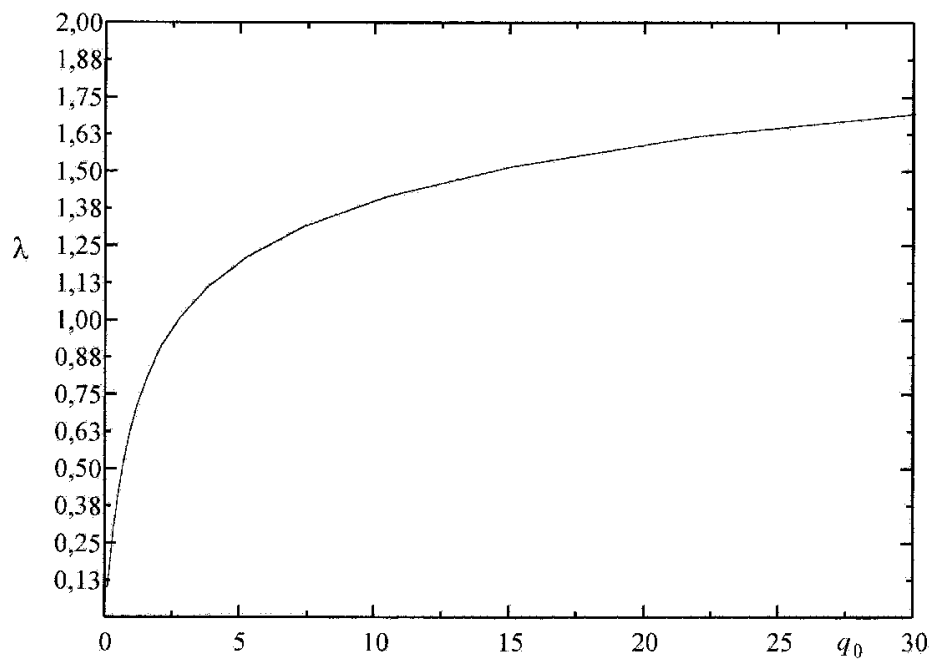

Figure $1-$ Behavior of $\lambda$ as a function of $q_{0}$.

Looking at Figures 2, 3 and 4, we see that the temperature distribution $t_{2}$ reaches to a minimum value which is smaller than the limit value $t_{0}$ that the function reaches at $+\infty$, i.e. the initial temperature, although in Figure 2 the function has no such minimum value. We shall find the values of the coefficient $L_{u}$ for which the function $T_{2}$ has a minimum value which is smaller than its limit value when $\eta \rightarrow+\infty$.

For $L_{u} \neq 1$, we take $T_{2}(\eta)$ for any $\eta>\lambda$, and we have

$$
T_{2}^{\prime}(\eta)=\frac{\varepsilon K_{o} L_{u}}{L_{u}-1}\left[\frac{\frac{2}{\sqrt{\pi L u}} e^{\left(-\frac{\eta^{2}}{L_{u}}\right)}}{1-\operatorname{erf}\left(\frac{\lambda}{\sqrt{L_{u}}}\right)}-\frac{\frac{2}{\sqrt{\pi}} e^{\left(-\eta^{2}\right)}}{1-\operatorname{erf}(\lambda)}\right]-\frac{\frac{2}{\sqrt{\pi}} e^{\left(-\eta^{2}\right)}}{1-\operatorname{erf} \lambda}
$$

and we get that

$$
T_{2}^{\prime}(\eta)=0 \Leftrightarrow \frac{\varepsilon K_{o} \sqrt{L_{u}}}{L_{u}-1} \frac{e^{\left(-\frac{\eta^{2}}{L_{u}}\right)}}{1-\operatorname{erf}\left(\frac{\lambda}{\sqrt{L_{u}}}\right)}=\left(\frac{\varepsilon K_{o} L_{u}}{L_{u}-1}+1\right) \frac{e^{\left(-\eta^{2}\right)}}{1-\operatorname{erf} \lambda}
$$

$\Leftrightarrow \eta$ is the solution of the following equation: 


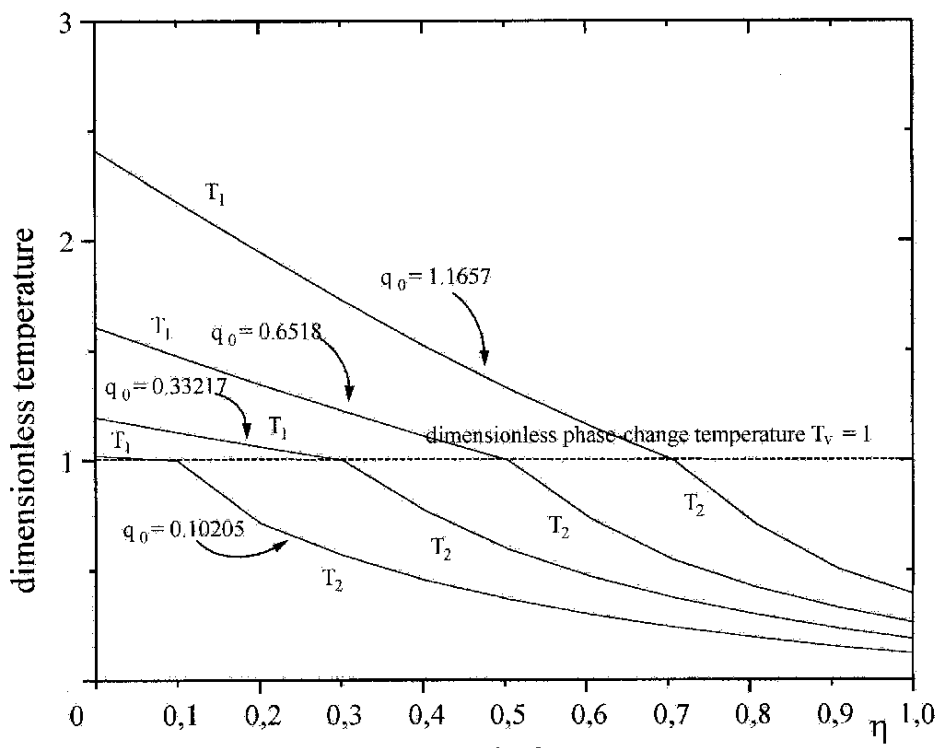

Figure 2 - Behavior of the temperature with respect to the dimensionless variable $\eta$ considering $L u=0.1<1$.

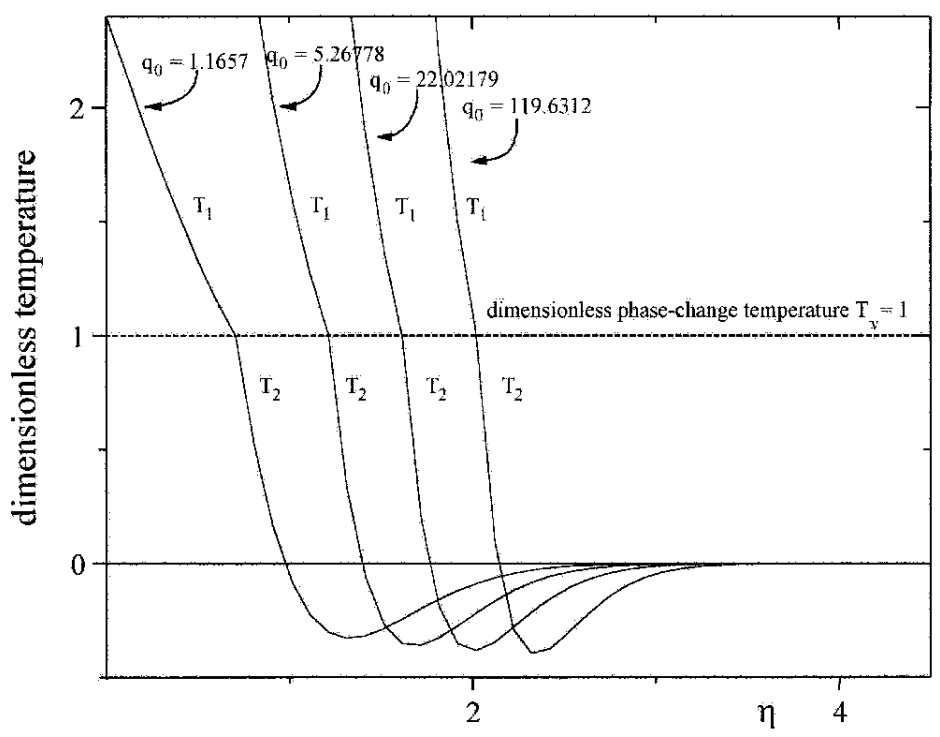

Figure 3 - Behavior of the temperature with respect to the dimensionless variable $\eta$ considering $L u=1$. 


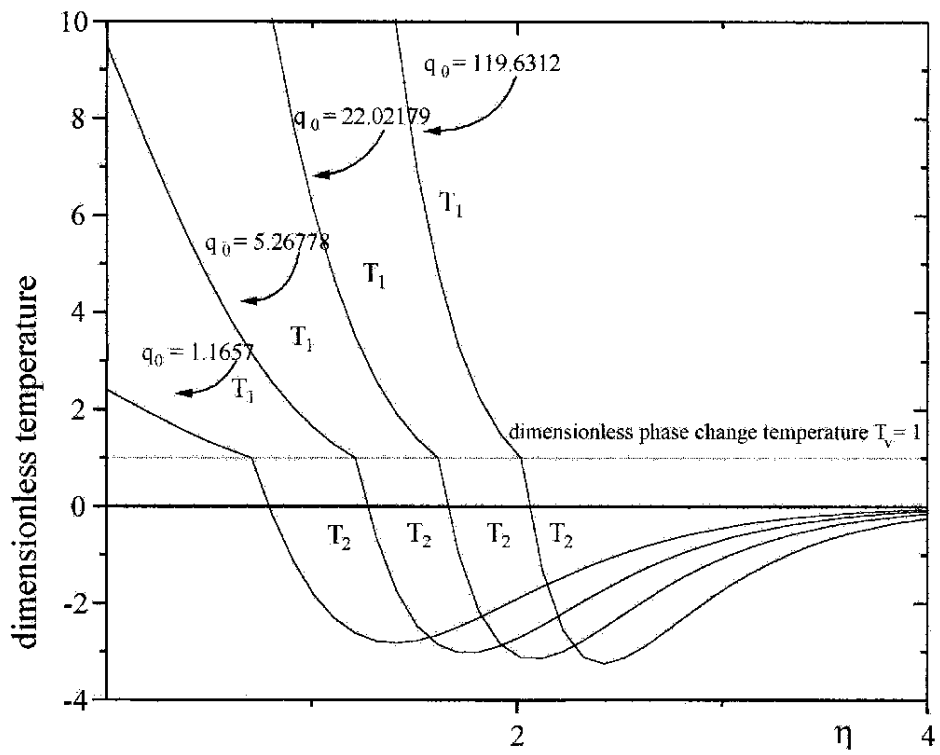

Figure 4 - Behavior of the temperature with respect to the dimensionless variable $\eta$ considering $L u=4>1$.

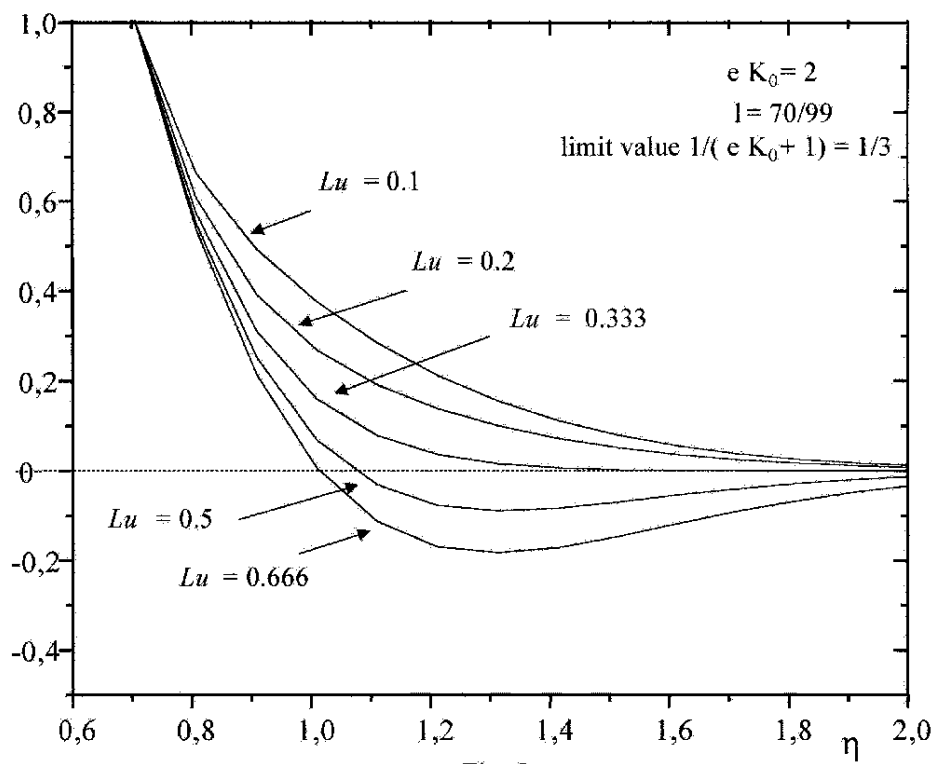

Figure 5 - Behavior of the temperature $T_{2}$ with respect to the dimensionless variable $\eta$ varying the values of $L u$. 


$$
S(x)=Z(x), x>\lambda,
$$

where $S$ and $Z$ are defined by:

$$
\begin{aligned}
& S(x)=\frac{\varepsilon K_{o} \sqrt{L_{u}}}{L_{u}-1} \frac{e^{\left(-\frac{x^{2}}{L_{u}}\right)}}{1-\operatorname{erf}\left(\frac{\lambda}{\sqrt{L_{u}}}\right)} \\
& Z(x)=\left(\frac{\varepsilon K_{o} L_{u}}{L_{u}-1}+1\right) \frac{e^{\left(-x^{2}\right)}}{1-\operatorname{erf} \lambda}
\end{aligned}
$$

Obviously, both $S$ and $Z$ are strictly decreasing (increasing) functions for any $x>0$ when $L_{u}>1\left(0<L_{u}<1\right)$. Moreover, we have

$$
\begin{aligned}
S(x)=Z(x) \Leftrightarrow \varepsilon K_{o} \sqrt{L_{u}} \frac{e^{\left(-\frac{x^{2}}{L_{u}}\right)}}{1-\operatorname{erf}\left(\frac{\lambda}{\sqrt{L_{u}}}\right)}=\left(\left(\varepsilon K_{0}+1\right) L_{u}-1\right) \frac{e^{\left(-x^{2}\right)}}{1-\operatorname{erf} \lambda} \\
\Leftrightarrow e^{\left(1-\frac{1}{L_{u}}\right) x^{2}}=\frac{\left(\left(\varepsilon K_{0}+1\right) L_{u}-1\right)}{\varepsilon K_{o} \sqrt{L_{u}}} \frac{1-\operatorname{erf}\left(\frac{\lambda}{\sqrt{L_{u}}}\right)}{1-\operatorname{erf} \lambda}
\end{aligned}
$$

which implies that in order to solve the equation (5.1), firstly we must to assume that $\left(\left(\varepsilon K_{0}+1\right) L_{u}-1\right)>0$, that is

$$
L_{u}>\frac{1}{\varepsilon K_{0}+1}
$$

Secondly, if $L_{u}>1$ we must to impose that

$$
\frac{\left(\left(\varepsilon K_{0}+1\right) L_{u}-1\right)}{\varepsilon K_{o} \sqrt{L_{u}}} \frac{1-\operatorname{erf}\left(\frac{\lambda}{\sqrt{L_{u}}}\right)}{1-\operatorname{erf} \lambda}>1
$$

which is always satisfied taking into account that the error function is a strictly increasing function. Moreover, if $L_{u}<1$ we must to impose that

$$
\frac{\left(\left(\varepsilon K_{0}+1\right) L_{u}-1\right)}{\varepsilon K_{o} \sqrt{L_{u}}} \frac{1-\operatorname{erf}\left(\frac{\lambda}{\sqrt{L_{u}}}\right)}{1-\operatorname{erf} \lambda}<1
$$


which is satisfied for all $0<L_{u}<1$. Therefore, if the Luikov number $L_{u}$ verifies the condition (5.4) we obtain that the solution of the equation $S(x)=Z(x)$ is given by

$$
\eta=\sqrt{\left(\frac{L_{u}}{L_{u}-1}\right) \log \left(\frac{\left(\left(\varepsilon K_{0}+1\right) L_{u}-1\right)}{\varepsilon K_{o} \sqrt{L_{u}}} \frac{1-\operatorname{erf}\left(\frac{\lambda}{\sqrt{L_{u}}}\right)}{1-\operatorname{erf} \lambda}\right)}
$$

Then we have obtained the following result:

Theorem 5.1. If the Luikov number $L_{u}$ verifies the condition (5.4) the temperature distribution $t_{2}$ reaches to a minimum value which is smaller than the initial temperature or its limit value at $+\infty$. The minimum value is attained when the dimensionless variable $\eta$ takes the value (5.7).

Remark 2. For large Luikov number the temperature distribution $t_{2}=t_{2}(\eta)$ has an absolute minimum value less than its initial temperature. Moreover, the minimum value for the Luikov number in order to have that property is given explicitely by the coefficient $\frac{1}{\varepsilon K_{0}+1}$, which is not an intuitive result.

\section{Conclusion}

Exact solutions for the problem of drying with coupled phase change in a porous medium with a heat flux condition on $x=0$ of the type $-\frac{q_{0}}{\sqrt{\tau}}$, with $q_{0}>0$, for any value of $L_{u}$ is obtained. This solution is only obtained when $q_{0}$ verifies a certain explicited inequality. The temperatures of the two phases and the mass-transfer potential were obtained by using the similarity method. Some illustrative results are shown. Finally, for large Luikov number (more precisely, $\left.L_{u}>\frac{1}{\varepsilon K_{0}+1}\right)$ we obtain that the temperature distribution $t_{2}$ reaches to an absolute minimum value which is smaller than the initial temperature (or its limit value at $+\infty$ ), and we characterize the coordinate of this point when the dimensionless variable $\eta=\frac{x}{2 \sqrt{a_{1} \tau}}$ takes the value (5.7) as a function of the data. 


\section{Acknowledgments}

This paper has been partially sponsored by the project "Free Boundary Problems for the Heat-Diffusion Equation' from CONICET-UA, Rosario (Argentina) and " Partial Differential Equations and Numerical Optimization with Applications" from Fundación Antorchas (Argentina).

\section{Nomenclature:}

$\begin{array}{ll}a_{i}, i=1,2 & \text { thermal diffusivity of the phase- } i . \\ a_{12} & \text { ratio of thermal diffusivities from phase } 1 \text { to phase } 2 \\ a_{m} & \text { moisture diffusivity } \\ c_{m} & \text { specific mass capacity } \\ c_{2} & \text { specific heat capacity } \\ k_{i}, i=1,2 & \text { thermal conductivity of the phase- } i . \\ k_{21} & \text { ratio of thermal conductivity from phase } 2 \text { to phase } 1 \\ K_{0}=\frac{L c_{m}\left(u_{0}-u_{v}\right)}{c_{2}\left(t_{v}-t_{0}\right)} & \text { Kossovitch number } \\ L & \text { latent heat of evaporation of liquid per unit mass } \\ L_{u}=a_{m} / a_{1} & \text { Luikov number } \\ q_{0} & \text { coefficient that characterizes the heat flux at } x=0 \\ s(\tau) & \text { position of the evaporation front } \\ t_{i}(x, \tau), i=1,2 & \begin{array}{l}\text { temperature of the phase- } i . \\ t_{0}\end{array} \\ t_{v} & \begin{array}{l}\text { initial temperature } \\ \text { temperature at the phase-change state }\end{array} \\ T_{i}, i=1,2 & \text { non-dimensional temperature of the phase- } i \\ u & \text { mass-transfer potential } \\ u_{0} & \text { initial mass-transfer potential } \\ U_{i}, i=1,2 & \text { dimensionless mass-transfer potential of the phase- } i \\ x & \text { dimensionless length }\end{array}$




\section{Greek symbols}

$\begin{array}{ll}\varepsilon & \text { coefficient of internal evaporation } \\ \eta & \text { dimensionless variable } \\ \lambda & \text { dimensionless constant which characterizes the evaporation front } \\ \rho_{m} & \text { density of moisture } \\ \tau & \text { time }\end{array}$

\section{Subscripts}

$\begin{array}{ll}0 & \text { at initial time, } t=0 \\ 1 & \text { dried porous medium, } 0<x<s(\tau) \\ 2 & \text { humid porous medium, } x>s(\tau) \\ v & \text { at evaporation front, } x=s(\tau)\end{array}$

\section{REFERENCES}

[1] A. Ali Cherif, A. Daïf, Etude numérique du transfert de chaleur et de masse entre deux plaques planes verticales en présence d'un film de liquide binaire ruisselant sur l'une des plaques chauffée, Int. J. Heat and Mass Transfer 42 (1999), 2399-2418.

[2] Y. Le Bray and M. Prat, Three-dimensional pore network simulation of drying in capillary porous media, Int. J. Heat and Mass Transfer 42 (1999), 4207-4224.

[3] H.S. Carslaw and J.C. Jaeger, Conduction of heat in solids, Clarendon Press, Oxford, (1959).

[4] J. Chen and J. Lin, Thermocapillary effect on drying of a polymer solution under non-uniform radiant heating, Int. J. Heat and Mass Transfer 43 (2000), 2155-2175.

[5] S.H. Cho, An exact solution of the coupled phase change problem in a porous medium, Int. J. Heat and Mass Transfer 18 (1975), 1139-1142.

[6] S.H. Cho and J.E. Sunderland, Heat conduction problem with melting or freezing, J. Heat. Transfer 91 (1969), 421-426.

[7] A. Fasano, M. Primicerio and D.A. Tarzia, Similarity solutions in class of thawing processes, Math. Models Methods Appl. Sci., 9 (1999), 1-10.

[8] C. Figus, Y. Le Bray, S. Bories and M. Prat, Heat and mass transfer with phase change in a porous structure partially heated: continuum model and pore network simulations, Int. J. Heat and Mass Transfer 42 (1999), 2557-2569.

[9] A.M. Gonzalez and D.A. Tarzia, Determination of unknown coefficients of a semi-infinite material through a simple mushy zone model for the two phase Stefan problem, Int. J. Engng. Sci. 34 (1996), 799-817. 
[10] L.N. Gupta, An approximate solution to the generalized Stefan's problem in a porous medium, Int. J. Heat Transfer 17 (1974), 313-321.

[11] J. Häger, M. Hermansson and R. Wimmerstedt, Modeling steam drying of a single porous ceramic sphere: experiments and simulations, Chem. Eng. Sci. 52 (1997), 1253-1264.

[12] A.L. Lombardi and D.A. Tarzia, Similarity solutions for thawing processes with a heat flux condition at the fixed boundary, Meccanica, 36 (2001), 251-264.

[13] A.V. Luikov, Heat and mass transfer in capillary-porous bodies, Adv. Heat Transfer 1 (1964), 123-184.

[14] A.V. Luikov, Heat and mass transfer in capillary-porous bodies, Pergamon Press, Oxford, (1966).

[15] A.V. Luikov, Analytical heat diffusion theory, Academic Press, New York, (1968).

[16] A.V. Luikov, Systems of differential equations of heat and mass transfer in capillary porous bodies, Int. J. Heat Mass Transfer 18 (1975), 1-14.

[17] A.V. Luikov, Heat and mass transfer, MIR Publishers, Moscow, (1978).

[18] A. Mhimid, S. Ben Nasrallah, J.P. Fohr, Heat and mass transfer during drying of granular products - simulation with convective boundary conditions, Int. J. Heat and Mass Transfer 43 (2000), 2779-2791.

[19] P. Perré and I.W. Turner, A 3-D version of TransPore: a comprehensive heat and mass transfer computational model for simulating the drying of porous media, Int. J. Heat and Mass Transfer 42 (1999), 4501-4521.

[20] E.A. Santillan Marcus and D.A. Tarzia, Explicit solution for freezing of humid porous halfspace with a heat flux condition, Int. J. Eng. Sci. 38 (2000), 1651-1665.

[21] D.A. Tarzia, An inequality for the coefficient $\sigma$ of the free boundary $s(t)=2 \sigma \sqrt{t}$ of the Neumann solution for the two-phase Stefan problem, Quart. Appl. Math. 39 (1981), 491-497.

[22] D.A. Tarzia, Soluciones exactas del problema de Stefan unidimensional, Cuadern. Inst. Mat. B. Levi 12 (1985), 5-36.

[23] D.A. Tarzia, A bibliography on moving free-boundary problems for the heat diffusion equation. The Stefan and related problems (with 5869 references), MAT-Serie A, \# 2 (2000). See www.austral.edu.ar/MAT-SerieA/2(2000). 